\title{
TOMOGRAFÍA DE COHERENCIA ÓPTICA COMO MARCADOR PARA LAS ENFERMEDADES NEURODEGENERATIVAS DE ALZHEIMER Y PARKINSON
}

Coba Caizaluisa Victoria ${ }^{1}$, Paredes Cordóva Cristina $^{1}$, Rivera Santana Jorge ${ }^{1,2 *}$

DOI: $10.48018 /$ rmv.v32.11.4

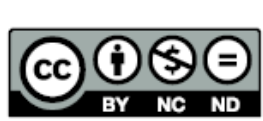

Este artículo está bajo una licencia de Creative Commons de tipo Reconocimiento - No comercial - Sin obras OPEN ACCESS derivadas 4.0 International.

1 Pontificia Universidad Católica del Ecuador. Facultad de Medicina. Carrera de Medicina. Quito - Ecuador.

2 Hospital Vozandes Quito - HVQ SA. Médico del servicio de Oftalmología. Quito - Ecuador.

ORCID ID:

Coba Caizaluisa Victoria

orcid.org/ 0000-0001-8683-5989

Paredes Cordóva Cristina

orcid.org/0000-0002-1357-8982

Rivera Santana Jorge

orcid.org/0000-0002-2117-2966

*Corresponding author: Rivera Santana Jorge E-mail: drjorgerivera@yahoo.com

Article history

Received: 16 - Sep - 2020

Accepted: 11 - Jan - 2021

Publish: 01 - Feb - 2021

Conflict of interest: All authors declared that there are no conflicts of interest.

Financial disclosure: The authors have no financial relationships relevant to this article to disclose.

Authors' contribution: All the authors contributed in the search, selection of articles and writing. All the authors reviewed and approved the final manuscript.

Forma de citar este artículo: Coba V, Paredes C, Rivera J. TOMOGRAFÍA DE COHERENCIA ÓPTICA COMO MARCADOR PARA LAS ENFERMEDADES NEURODEGENERATIVAS DE ALZHEIMER Y PARKINSON. Rev Med Vozandes. 2021; 32 (1): .....- .......

\section{Resumen}

\section{Objetivo}

La Tomografía de Coherencia Óptica (OCT) es un examen de gabinete utilizado de manera complementaria en patología de la retina. Este trabajo tiene como objetivo llevar a cabo una revisión bibliográfica sobre el examen de OCT como biomarcador de las enfermedades neurodegenerativas, como el Alzheimer y Parkinson a través de un análisis cualitativo de artículos médicos publicados entre los años 2015 y 2019.

\section{Método \\ Se realizó una revisión sistemática retrospectiva sin metaanálisis de la literatura según un diseño de investigación observacional que resume los resultados de varias investigaciones primarias. Se seleccionaron diez estudios sobre la aplicación de OCT y angio-OCT, en pacientes adultos mayores de 60 años con enfermedad de Alzheimer preclínica (EA) y de enfermedad de Parkinson (EP) quienes presentaban en la retina anormalidades microvasculares.}

\section{Resultados}

La OCT muestra cambios dinámicos en las áreas de engrosamiento de la capa de células ganglionares y membrana plexiforme interna y capa de fibras nerviosas (GCIPL y NFL, respectivamente, por sus siglas en inglés), en la mácula adyacente durante el desarrollo de EA, mientras que en las primeras etapas de la EP revela adelgazamiento de la retina, en relación con la gravedad de la enfermedad y con la degeneración de dopamina nigral.

\section{Conclusiones}

La Tomografía de Coherencia Óptica es un potencial biomarcador para EA y EP, y ante la sospecha temprana de estas patologías podría solicitarse como apoyo diagnóstico.

Palabras clave: enfermedades neurodegenerativas, Alzheimer, Parkinson, tomografía de coherencia óptica.

\section{Abstract}

OPTICAL COHERENCE TOMOGRAPHY AS A MARKER FOR NEURODEGENERATIVE ALZHEIMER'S AND PARKINSON'S DISEASE

\section{Background}

Optical coherence tomography (OCT) is an ancillary test used in retinal pathology. The objective of the present dissertation is to review literature regarding (OCT) and its importance as a biomarker in neurodegenerative Alzheimer's and Parkinson's disease, through an analysis of medical journal articles published between 2015 and 2019.

\section{Methods}

A retrospective systematic review without meta-analysis of literature was carried out using observational research design, allowing to summarize the 
Keywords: neurodegenerative diseases, Alzheimer's disease, Parkinson's disease, optical coherence tomography. results of multiple primary investigations. Ten studies published between 2015 and 2019 regarding the application of OCT and ANGIO OCT in adult patients older than 60 years of age with preclinical Alzheimer's disease (AD) or Parkinson's disease (PD) with retinal microvascular abnormalities, were selected

\section{Results}

Areas of increased thickness of ganglion cell-inner plexiform layer (GCIPL) and nerve fiber layer (NFL) adyacent to the macula suggest that dynamic changes can occur as a result of AD progression. Thinning of the retina is present during early stages of PD. This correlates with disease severity and may be related to degeneration of dopaminergic neurons in the substantia nigra.

\section{Conclusion}

Optical Coherence Tomography is a potential biomarker for AD and PD, and if these pathologies are suspected early, and if these pathologies are suspected early, it could be requested as diagnostic support

\section{Introducción}

Teniendo evidencia de la utilidad de la OCT, se ha buscado implementarla en el estudio de varios trastornos neurológicos, el más común de estos en todo el mundo es la demencia y se estima que 1 de cada 3 de los nacidos en países desarrollados presentará esta patología. La enfermedad de Alzheimer es la patología más frecuente en este grupo y se caracteriza principalmente por la acumulación de amiloidebeta $(A \beta)$ y proteína tau en estructuras del cerebro. Es una enfermedad irreversible(1).

El diagnóstico definitivo de EA se establece postmortem al detectar en la autopsia en el tejido cerebral ovillos neurofibrilares y placas de acumulo de proteína $A \beta^{(2)}$. Teniendo en cuenta que la fase clínica de la enfermedad progresa sin una clara temporalidad, el costo que cada individuo representa para los sistemas de salud es elevado.

La enfermedad de Parkinson (EP) es considerada dentro de las enfermedades neurodegenerativas la segunda en frecuencia; es un trastorno del movimiento progresivo cuyas características son bradicinesia, rigidez muscular, temblor en reposo, y pérdida de reflejos posturales; se asocia con complicaciones cognitivas que incluyen: demencia, depresión, trastornos del sueño y psicosis. Dentro de la fisiopatología de la enfermedad, encontramos una pérdida de neuronas dopaminérgicas en la sustancia negra y en otras áreas del cerebro ${ }^{(3)}$.

Si tanto las enfermedades neuro degenerativas de Alzheimer y Parkinson así como el ojo, específicamente la retina, pues ésta es una extensión del sistema nervioso central, poseen los mismos mecanismos patogénicos como: estrés oxidativo, daño mitocondrial, excitotoxicidad por glutamato, procesos de agregación proteica y activación glial, se puede considerar al órgano de la visión como un órgano blanco ${ }^{(3)}$.

El buscar manifestaciones oculares en patologías cerebrales parece razonable, se han descubierto varios cambios oculares observados en evaluaciones oftalmológicas en pacientes con enfermedades del sistema nervioso central, tales como Alzheimer, ictus, esclerosis múltiple y Parkinson. En diversos de estos trastornos, las expresiones oculares anteceden a menudo a los síntomas cerebrales, lo que parece revelar que las exploraciones oculares podrían brindar un medio de diagnóstico precoz ${ }^{(4)}$.
La capa de fibras nerviosas y las células ganglionares que forman parte de la retina se consideran biomarcadores indirectos del sistema nervioso central debido a que comparten un mismo origen embriológico. En 1989, gracias al estudio realizado por Blanks y colaboradores, se demostró que pacientes con EA presentan cambios microscópicos en la retina debido a la degeneración en las células ganglionares ${ }^{(5)}$. Esto ha permitido predecir hallazgos cerebrales patológicos en los pacientes que sufren determinadas enfermedades neurológicas.

En pacientes afectados de Alzheimer que se realizaron estudios de OCT, se observa una reducción significativa del espesor de la capa de fibras nerviosas de la retina ${ }^{(6)}$.

\section{Justificación}

Cada día se incrementan los esfuerzos para conseguir una detección precoz del deterioro cognitivo y brotan en el panorama científico entidades diagnósticas como el deterioro cognitivo leve (DCL) y las quejas subjetivas de memoria (QSM). Es por ello que aparecen nuevos y numerosos biomarcadores estudiados para conseguir dicho objetivo, entre ellos, la Tomografía de Coherencia Óptica.

Se ha realizado un estudio que utiliza la OCT para medir el grosor macular y la capa de fibras nerviosas de la retina en pacientes diagnosticados de EA, pacientes con $D C L$, en individuos con QSM y en sujetos control. Gracias a esto, se ha descubierto que existen diferencias estadísticamente significativas en cuanto al grosor macular entre todos los grupos estudiados

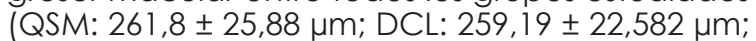
EA leve: $258,53 \pm 14,804 \mu \mathrm{m}$; EA moderada: 249,32 $\pm 18,467 \mu \mathrm{m})$ y sujetos control $(271,96 \pm 15,57 \mu \mathrm{m})$. Respecto a la capa de fibras nerviosas de la 
retina, ocurre lo mismo, y la diferencia es estadísticamente significativa frente al grupo control $(94,51 \pm 9,203 \mu \mathrm{m}) \mathrm{de}$ todos los grupos (QSM: 90,44 $\pm 9,059 \mu \mathrm{m}$; DCL: 89,4 $\pm 10,421$ $\mu \mathrm{m}$; EA leve: $87,12 \pm 10,279 \mu \mathrm{m}$; EA moderada: $82,25 \pm 10,636$ um). (Aguirán Esquej, 2017, pág. 35)

Es por todo lo referenciado por este autor que puede decirse que la OCT es una herramienta que sirve de apoyo y biomarcador que facilitan el diagnóstico precoz del DCL y de la EA.

En esta investigación se toman en cuenta dos de las principales enfermedades neurodegenerativas que existen. Por una parte, EA que es la enfermedad más frecuente de esta categoría y cuyos criterios de diagnóstico clínico no tienen un nivel de certeza que logre diferenciar esta enfermedad con otras causas de demencia. Por otro lado, EP la cual presenta afectaciones del movimiento de quien la padece, provocadas por la degeneración y/o muerte de células neuronales. Estas dos patologías tienen un impacto importante en la calidad de vida de los pacientes con estas enfermedades y de su entorno cercano.

Existen estudios que han evidenciado la utilidad de la OCT en el diagnóstico de cambios neurodegenerativos, especialmente en aquellos relacionados a la función cognitiva. Contar con métodos no invasivos y tempranos de diagnóstico es clave en la medicina contemporánea, especialmente para enfermedades que generen alto impacto a los sistemas de salud.

Tabla 1: Términos de referencia para la búsqueda científica

Término Principal

Coherence Tomography, Optical

OCT Tomography

Tomography, OCT

Optical Coherence Tomography
Conector

AND / OR

AND / OR
Coherence Tomography, Optical

OCT Tomography

Tomography, OCT

Optical Coherence Tomography
Término secundario

Alzheimer's Disease

Dementia, Senile

Senile Dementia

Dementia, Alzheimer Type

Alzheimer Type Dementia

Alzheimer-Type Dementia (ATD)

Alzheimer Type Dementia (ATD)

Dementia, Alzheimer-Type (ATD)

Alzheimer Type Senile Dementia

Alzheimer Dementia

Alzheimer Dementias

Dementia, Alzheimer

Dementias, Alzheimer

Senile Dementia, Alzheimer Type

Acute Confusional Senile Dementia

Senile Dementia, Acute Confusional

Dementia, Presenile

Presenile Dementia

Alzheimer Disease, Late Onset

Late Onset Alzheimer Disease

Alzheimer's Disease, Focal Onset

Focal Onset Alzheimer's Disease

Familial Alzheimer Disease (FAD)

Alzheimer Disease, Familial (FAD)

Alzheimer Diseases, Familial (FAD)

Familial Alzheimer Diseases (FAD)

Alzheimer Disease, Early Onset

Early Onset Alzheimer Disease

Presenile Alzheimer Dementia

Idiopathic Parkinson's Disease

Lewy Body Parkinson's Disease

Parkinson's Disease, Idiopathic

Parkinson's Disease, Lewy Body

Parkinson Disease, Idiopathic

Parkinson's Disease

Idiopathic Parkinson Disease

Lewy Body Parkinson Disease

Primary Parkinsonism

Parkinsonism, Primary 


\section{Diagrama de flujo PRISMA}

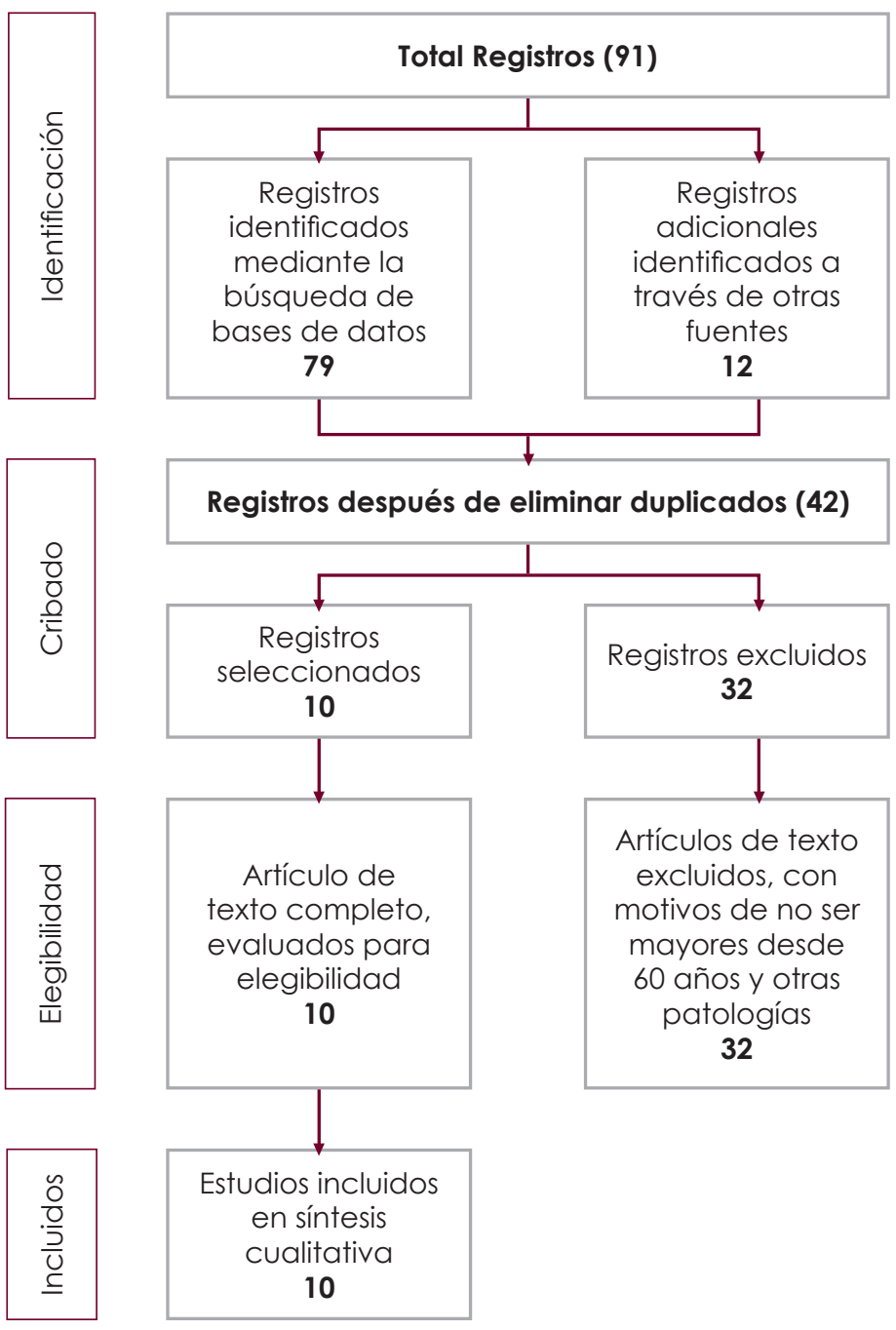

Fuente: Tripdatabase, MEDLINE, EMBASE, Excerpta Medica, Cochrane Library (2020) Realizado por: Autores

La OCT es una tecnología que tiene evidencias sobre su eficacia en Parkinson y en Alzheimer, por lo que en este estudio se realizará una revisión sistemática sin metaanálisis de investigaciones disponibles que permitan establecer esta conclusión.

\section{Método}

Este es un trabajo de titulación modalidad revisión sistemática sin metaanálisis basado en la recolección de datos de estudios publicados del 2015-2019, no se contará con información individual de pacientes, tampoco con contacto directo con los mismos por lo que no se usó consentimiento informado.

\section{Selección de la estrategia de búsqueda}

La búsqueda de literatura se realizó en datos bibliográficos computarizados de estudios publicados en revistas indexadas (MEDLINE, EMBASE, Excerpta Medica, Cochrane Library,
Tripdatabase.), con los siguientes términos incluidos, donde constan estudios pertinentes en revistas de alto impacto y Science Citation Index de las áreas de medicina y epidemiología para los estudios controlados aleatorizados (ECA) publicados en los últimos 5 años. Con fecha de última búsqueda de los árticulos a utilizar 30 de junio del 2020.

Estrategia de Búsqueda: Los términos incluidos fueron: (OCT) Tomografía de coherencia óptica, (OCT) Optical coherence tomography, (EA) Enfermedad de Alzheimer, (AD) Alzheimer's disease, (EP) Enfermedad de Parkinson, (PD) Parkinson's disease en español e inglés.

\section{Selección de estudios}

Se incluirán estudios cuya población se limite a Enfermedad de Parkinson (EP) y Enfermedad de Alzheimer (EA) con relación a la utilización de OCT como marcador de estas enfermedades. La elegibilidad y criterio de inclusión de los artículos se refirieron a que los trabajos sean catalogados como artículos de investigación original que proporcionen una cantidad de pacientes que se pueda extrapolar con las enfermedades de Alzheimer, Parkinson y la eficacia del empleo de la Tomografía de Coherencia Óptica. Se admitieron tantos ensayos controlados que sean aleatorizados como estudios observacionales. También se realizó control de calidad para comparar los datos resumidos con los artículos originales y evitar duplicados. Se obtuvieron 79 resultados en total: 12 se encontraron en tesis de grado y de doctorado, 42 artículos fueron muy relevantes para la investigación bibliográfica realizada de los cuales 32 fueron excluidos por diversas razones: resultados no acordes para los objetivos de este trabajo, edad de la población fuera del rango del adulto mayor, estudios solo basados en generalidades de la EA y EP, estudio de pacientes con otro tipo de factores que no son parte del presente estudio, estudios con riesgo de sesgo que pudiesen afectar la evidencia acumulada demostrado con Newcastle - Ottawa Quality Assessment Scale. De la documentación antes mencionada se escogieron 10 artículos que contenían información relevante para ser incluida en esta investigación, como fueron: factores de riesgo, incidencia y prevalencia de EA y EP en los adultos mayores de 60 años tanto en mujeres como en hombres, presentación clínica de la enfermedad y un diagnóstico a través de la tomografía.

\section{Extracción de datos}

La extracción de datos fue realizada utilizando un formulario estandarizado. Se extrajeron los siguientes datos: autores, revista, título, año de publicación, lugar. (Tabla 2) 
Tabla 2: Estudios seleccionados

\section{Autores}

1 O' Bryhim, Elizabeth; Kung, Nathan, Coble, Dean7

2 Santos, Claudia; Johnson, Lenworth; Sinoff; Stuart; Festa, Elena; heindel, William; Snyder, Peter8

3 Lad, Eleonora; Mukherjee, Dibyendu; Stinnett, Sandra; Cousins, Scott; Potter, G; Burke; Farsiu, Sina; Whistson, Heather9

4 Bulut, Mehmet; Kurtulus, Fatma; Gozakaya, Onursal; Erol, Kasim; Cengis, Ayse; Melih, Akidan; Aylin, Yaman 10

5 Maylankody, Pooja; Battu, Rajani; Khanna, Anjani; Lenka, Abhishek; yadavv, Ravi; Kumar, Pramod 11

6 Alida van de Kreeke, Jacoba; Nguyen, Hoang-Ton; Verbraak, Frank; Visser, Jelle; Pieter 12

7 Saute, María; Obis, Javier; Rodrigo, María J; Otin, Sofía; Fuentes, María I; Vilades, Elisa 13

8 Nunes, Ana; Silva, Gilberto, Duque, Cristina; Januario, cristina; Santana, Isabel; Ambrosio, Antonio Francisco, Castelo-Branco, Miguel; Bernardes, Ruil4

9 Ahn, Jeeyun; Lee, Jee-Young; Yoon, Jin; Eun; Kim, Jong 15

10 Cunha, Provetti; Leonardo; Maciel Almeida, Ana Laura; Costa, ferreira; Carolina; Monteiro, Mariol6

\section{Revista Título Publicación}

JAMA*

Network

ELSEVIER

PLOS ONE

Association of Preclinical Alzheimer

Disease with Optical Coherence Tomographic Angiography Findings

Change in retinal structural anatomy during the preclinical stage of Alzheimer's disease.

Evaluation of inner retinal layers as biomarkers in mild cognitive impairment to moderate Alzheimer's disease

PLOS ONE

Evaluation of optical coherence tomography angiographic findings in Alzheimer's type dementia

\section{ELSEVIER}

Optical coherence tomography as a tool to evaluate retinal changes in Parkinson's disease

Open Access

Journal of Ophtalmology

PLOS ONE

Optical coherence tomogra-

phy angiography in preclinical Alzheimer's disease

Optical Coherence Tomography as a Biomarker for Diagnosis, Progression, and Prognosis of Neurodegenerative Diseases

Retinal texture biomarkers may help to discriminate between Alzheimer's, Parkinson's, and healthy controls

Related

Article

Open Access
Retinal thinning associates with nigral dopaminergic loss in the novo Parkinson disease

The role of optical coherence tomography in Alzheimer's disease
Año Lugar

2018 Missouri, Estados Unidos

2018 North Carolina Estados Unidos

2018 North Carolina, Estados Unidos

2017 Turkey

2015 Karnataka, India

2019 Amsterdam, Holanda

2016 Zaragoza, España

2019 Combria, Portugal

2018 South Korea

2016 Brasil

Nota. *Journal of the American Medical Association

Fuente: JAMA Network, PLOS ONE, Journal of Ophtalmology, ELSEVIER (06/2020)

Realizado por: Autores

\section{Resultados}

Después de extraerlainformación delosestudiosrelacionados con la Tomografía de Coherencia Óptica como marcador temprano para las enfermedades de Alzheimer y Parkinson y partiendo de una revisión sistemática sin metaanálisis exhaustiva, se realizó una tabla descriptiva de los hallazgos más importantes en cada estudio, ver (Anexo A)

En el estudio realizado por O'Bryhim et al.(7), las mediciones automatizadas del grosor de la capa de fibra nerviosa de la retina, el de la capa de células ganglionares, el grosor foveal interno y externo, la densidad vascular, el volumen macular y la zona avascular foveal se obtuvieron utilizando un sistema angio- OCT de los ojos de todos los participantes. Se analizaron cincuenta y ocho ojos de 30 participantes $153 \%$ mujeres; edad media de 74.5 años; rango de edad, 62-92 años) se incluyeron en el análisis. Un participante era afroamericano y 29 eran blancos. Catorce participantes tenían biomarcadores positivos para EA y, por lo tanto, un diagnóstico de EA preclínica (edad media de 73,5 años); 16 
sin biomarcadores sirvieron como grupo de control (edad media de 75,4 años). La zona avascular foveal aumentó en el grupo de biomarcadores positivos en comparación con los controles (media 0.364 [0.095] frente a 0.275 [0.060] mm2; P = .002). El grosor foveal interno medio disminuyó en el grupo de biomarcadores positivos $(66,0 \text { frente a 75,4 } \mu \mathrm{m} ; \mathrm{p}=0,03)^{(7)}$.

Cincuenta y seis adultos mayores (edad media $=65.36$ años) con múltiples factores de riesgo para EA completaron la tomografía de coherencia óptica del dominio espectral por imágenes retinianas y pruebas cognitivas al inicio del estudio. Veintisiete meses después, completaron los mismos exámenes y un estudio de tomografía por emisión de positrones 18F-florbetapir.

En comparación con los sujetos sanos de control, aquellos en la etapa preclínica de EA mostraron una disminución significativa en el volumen de la capa de fibra nerviosa retiniana macular (mRNFL) durante un período de seguimiento de veinte y siete meses, así como también una disminución en la capa nuclear externa e interna volúmenes y grosor de la capa plexiforme en cuadrante inferior. (Garcia, 2019). Además, se observa una relación de la información visual y también de la auditiva percibidas del habla, esta relación fue significativa con la extensión de la reducción del volumen de mRNFL. Se observa una marcada baja en los volúmenes de mRNFL, capa nuclear externa y plexiforme interna, en la preclínica de la EA en relación con los controles. Además, la pérdida axonal en gran medida mielinizada en el RNFL está relacionada con el aumento de la acumulación de amiloide- $\beta$ neo-cortical después de controlar la edad.

La pérdida de volumen en el RNFL, durante la etapa preclínica, no está relacionada con el rendimiento en medidas de memoria episódica o resolución de problemas. Sin embargo, este cambio en la retina parece estar ligeramente relacionada con disminuciones relativas en el rendimiento en una medida de eficiencia de integración audiovisual que se ha avanzado recientemente como un posible marcador cognitivo temprano de deterioro cognitivo leve.

Un estudio transversal (9) de casos y controles incluyó a quince pacientes con deterioro cognitivo leve (DCL), quince pacientes con EA leve a moderada y dieciocho adultos cognitivamente normales ${ }^{(9)}$. Los espesores de NFL y GCIPL en la OCT se midieron utilizando el programa de análisis de retina de tomografía de coherencia óptica Duke (DOCTRAP) y el software Spectralis. Se demostró que los espesores regionales de NFL o GCIPL en OCT maculares o nerviosos no diferían entre los grupos. Sin embargo, un análisis de regresión multivariante identificó áreas maculares con un engrosamiento o adelgazamiento significativo en NFL y GCIPL en pacientes con DCL y EA. Los hallazgos primarios controvirtieron los informes anteriores de NFL más delgada en EA moderada a severa (9). Las áreas de engrosamiento de GCIPL y NFL en la mácula adyacente a las áreas de adelgazamiento, como lo revela un modelo estadístico más complejo, sugieren que NFL y GCIPL pueden sufrir cambios dinámicos durante la progresión de EA.

Estudio realizado por Bulut et al. ${ }^{(10)}$, incluyó veintiséis pacientes en el grupo de casos y veintiséis sujetos de edad y sexo similar en el grupo de control. Se realizó un examen oftalmológico y neurológico detallado para todos los sujetos incluidos en el estudio. La retina, las estructuras vasculares coroidales y el grosor coroideo (TC) de todos los sujetos se analizaron de manera detallada con un dominio espectral comercial angio-OCT. Además, todos los participantes se sometieron a un examen neurológico detallado que incluyó la prueba del Mini Examen del Estado Mental (MMSE) para evaluar la función cognitiva.

En el grupo de pacientes medidos con el dispositivo de alta modulación, la puntuación MMSE fue significativamente menor que la del grupo control ( $p<0,001)$. La densidad vascular retiniana fue significativamente menor que la del grupo control en todas las zonas ( $p<0.05$ ). La zona avascular foveal (FAZ) se amplió significativamente en comparación con el grupo control ( $p=0,001)$. La TC fue significativamente menor en el grupo de pacientes con ATD ( $P$ $<0.001)$. Las tasas de flujo externo de la retina y las coroides fueron más bajas en el grupo de pacientes con ATD, mientras que la diferencia no fue significativa ( $p>0.05)$. Además, se encontró una correlación significativa entre el MMSE y todos los parámetros de densidad vascular, parámetro TC y FAZ probados con imágenes OCTA $(p<0.05)^{(10)}$.

En el estudio de van de Kreeke et al.(12), los investigadores, evaluaron 124 individuos cognitivamente sanos (gemelos mono zigotos con edades entre 60 y 93 años) se sometieron a [18 F] tomografía por emisión de positrones (PET) con flutemetamol y OCTA. Las exploraciones de PET se clasificaron visualmente para la positividad cortical de la proteína amiloide-beta $(A \beta)$. El potencial de unión cortical global no desplazable paramétrico (BP ND) se utilizó como una medida continua para la agregación de $A \beta$. Se midieron el tamaño de la FAZ y las densidades de los vasos para el anillo interno y externo de la ETDRS macular y en un anillo de 3-6 mm alrededor de la cabeza del nervio óptico (ONH).

Las medidas de OCTA se asociaron con la puntuación visual $A \beta$, BP ND y la carga de amiloide estimada por la concordancia doble en la puntuación visual A $\beta$. Las correlaciones en los pacientes gemelos se estimaron como una medida de la heredabilidad máxima de las medidas OCTA. De los cuales 13 de 124 participantes fueron $A \beta+$. Los individuos $A \beta+$ tenían una densidad de vasos significativamente mayor que los individuos $A \beta-$ en todas las regiones, pero no diferían en el tamaño de la FAZ. Los análisis en gemelos mostraron una asociación positiva entre las densidades de vasos en todas las regiones. BP ND tendió a asociarse con una mayor densidad de vasos en el anillo interno. Las correlaciones en gemelos fueron moderadas / altas para todos los parámetros OCTA, excepto la densidad de los vasos alrededor de la $\mathrm{ONH}$, que se correlacionó débilmente ${ }^{(12)}$ 
Se calcularon imágenes del fondo de ojo a partir de datos de la OCT y analizaron la disposición estructural del tejido retiniano utilizando métricas de textura. Se crearon modelos de clasificación de clase clínica para distinguir entre controles sanos, EA y EP, utilizando el aprendizaje automático, mostrando los siguientes resultados:

La sensibilidad media es $88.7 \%, 79.5 \%$ y $77.8 \%$, para los ojos de los controles sanos, EA, EP, respectivamente. Cuando el mismo sujeto tiene la misma clasificación para ambos ojos, el $94.4 \%$ (mediana) de las clasificaciones son correctas ${ }^{(2)}$.

Las imágenes de OCT de la retina humana transmiten una cantidad significativa de información que discrimina entre múltiples estados neurodegenerativos, incluso cuando todavía no hay diferencias de grosor (14). En otro de los estudios escogidos se analizaron cuarenta y nueve pacientes con EP y cincuenta y cuatro controles pareados por edad fueron analizados. El examen oftalmológico y la tomografía de coherencia óptica de mácula se realizaron con microperimetría adicional, $\mathrm{N}$ - (3- [18F] fluoropropil) -2-carbometoxi-3- (4-yodofenil) nortropane PET, y las exploraciones de resonancia magnética se realizaron en pacientes con EP solamente. El grosor y el volumen de la capa retiniana se midieron en subcampos de los círculos de estudio de tratamiento temprano de la retinopatía diabética de 1, 2,22 y 3,45 mm y se compararon en pacientes con EP y controles. Además, se examinó la correlación del adelgazamiento de la capa interna de la retina con la respuesta micro perimétrica en pacientes con EP, y se analizaron las relaciones entre el grosor de la capa retiniana y las densidades del transportador de dopamina en el caudado ipsilateral, el putamen anterior y posterior y la sustancia negra. Así mismo se observó un adelgazamiento de la capa retiniana en los sectores temporales e inferiores de 2,22 mm ( $p<0,05$ falso ajustado por tasa de descubrimiento) de pacientes con EP sin tratamiento farmacológico, particularmente las capas de células ganglionares y plexiformes internas. El grosor de las capas GCIPL en el sector inferior de $2.22 \mathrm{~mm}$ mostró una relación negativa con la escala desarrollada por Melvin Yahr y Margaret Hoehn ( $p=0.032$ y 0.014 , respectivamente). También hubo correlación positiva entre la sensibilidad macular y el grosor de la capa retiniana en todos los sectores de $3.45 \mathrm{~mm}$, el sector superior de $2.22 \mathrm{~mm}$ y el círculo de $1 \mathrm{~mm}$ ( $p<0.05$ para todos). Hubo una asociación entre el adelgazamiento de la retina y la pérdida dopaminérgica en la sustancia negra izquierda (tasa de descubrimiento falsa ajustada $p<0,001$ ).

Mailankody et al.(11), realizó una evaluación prospectiva, basada en 30 pacientes con EP y 30 controles sanos. Varios parámetros tales como RNFL, espesor macular central (CMT), volúmenes maculares centrales y totales (TMV) y espesor retiniano fueron analizados usando $\mathrm{OCT}$, mostrando las siguientes conclusiones:

(a) El grosor de RNFL no fue significativamente diferente entre los pacientes y los controles. Se encontró una correlación negativa significativa entre el grosor RNFL en el sector nasal superior derecho y la puntuación motora UPDRS. (b) SeencontróquelaCMTseredujosignificativamente en el ojo derecho y se observó una correlación negativa con la puntuación motora UPDRS.

(c) TMV fue significativamente mayor en pacientes en comparación con los controles.

(d) Se descubrió que la capa externa de la retina en el cuadrante nasal derecho y los cuadrantes inferiores derechos eran significativamente más delgados en pacientes con EP.

\section{Discusión}

Dentro de los estudios seleccionados en 30 pacientes con biomarcadores positivos para EA tuvieron un incremento de la zona avascular foveal mientras que el grosor interno medio disminuyó; 56 adultos con múltiples factores de riesgo para EA se les aplicó OCT de dominio espectral por imágenes retinianas y pruebas cognitivas al inicio del estudio y veintisiete meses después se repitieron los mismos exámenes y un estudio de tomografía de positrones 18F-florbetaquir. En la zona macular de la retina cuya sensibilidad es mayor y es la encargada de la visión más fina es donde se observan los primeros cambios, como se demuestra en este estudio realizado a estos 30 pacientes. Cuando se compara estos resultados con los sujetos sanos de control, se muestra una disminución significativamente relacionable en el volumen de la capa de fibra nerviosa retiniana macular (mRNFL) así como un tamaño menor de la capa nuclear externa e interna volúmenes y grosor de la capa plexiforme en cuadrante inferior. De tal modo se demostró que la pérdida de volumen en el RNFL, durante la etapa preclínica, no está relacionada con el rendimiento en medidas de memoria episódica o resolución de problemas. Sin embargo, este cambio en la retina está ligeramente relacionada con disminuciones relativas en el rendimiento en una medida de eficiencia de integración audiovisual que se ha avanzado recientemente como un posible marcador cognitivo temprano de deterioro cognitivo leve. Se examinó la correlación del adelgazamiento de la capa interna de la retina en pacientes con EP, y se analizaron las relaciones entre el grosor de la capa retiniana y las densidades del transportador de dopamina en el caudado ipsilateral, el putamen anterior y posterior y la sustancia negra. Se observó un adelgazamiento de la capa retiniana en los sectores temporales e inferiores de $2,22 \mathrm{~mm}$ (p $<0,05$ falso ajustado por tasa de descubrimiento) de pacientes con EP sin tratamiento farmacológico, particularmente las capas de células ganglionares y plexiformes internas. El grosor de estas capas en el sector inferior de $2.22 \mathrm{~mm}$ mostró una correlación negativa con la etapa de Hoehn y Yahr $(p=0.032$ y 0.014, respectivamente). Se encontró que la CMT se redujo significativamente en el ojo derecho y se observó una correlación negativa con la puntuación motora UPDRS. TMV fue significativamente mayor en 
pacientes en comparación con los controles. Se descubrió que la capa externa de la retina en el cuadrante nasal derecho y los cuadrantes inferiores derechos eran significativamente más delgados en pacientes con EP.

Además, uno de los puntos fuertes de este artículo fue el uso de varios criterios para seleccionar los estudios. Por ejemplo, una vez aplicadas las estrategias de investigación, se seleccionaron diferentes artículos científicos bajo estrictos criterios metodológicos, epidemiológicos y estadísticos para garantizar el uso de los artículos disponibles que estaban de acuerdo con las necesidades y condiciones de este ensayo.

Sin embargo, para el presente estudio se necesitan más estudios y mayor número de muestra para corroborar nuestros hallazgos ya que los hallazgos no son extrapolables.

De dichos artículos se han extraído las siguientes conclusiones relevantes, establecidas en relación con los elementos fundamentales de cada investigación, como se muestra a continuación.

\section{Conclusiones}

Del estudio realizado se concluye que las personas cognitivamente sanas, mayores de 60 años, y con EA preclínica, presentan en la retina anomalías microvasculares, además de variaciones arquitectónicas; estas variaciones ocurren en etapas más tempranas de EA que las que se han demostrado previamente.

Con la tecnología OCT se ha podido reconocer cambios tempranos en la retina relacionados estrechamente con las patologías de EA y EP, permitiendo a futuro que el personal de salud pueda realizar un diagnóstico preclínico de las mismas.

En pacientes con EA las patologías vasculares retinianas y coroideas detectadas mediante imágenes de OCTA son evidentes, lo que convierte a este examen en un potencial biomarcador en el diagnóstico temprano de la enfermedad, el seguimiento de su progresión y el resultado del tratamiento farmacológico.

En pacientes diagnosticados con EA y EP Ia OCT revela un adelgazamiento en las capas GCIPL y NFL, manifestándose además cambios dinámicos durante la progresión de EA.

Los estudios de OCT realizados a pacientes diagnosticados con EA y EP, enfocados en los cambios del espesor CFNR, muestran una estrecha relación con la pérdida neuronal y axonal presente en estas enfermedades neurodegenerativas.

\section{Comentarios}

La revisión sistemática retrospectiva sin metaanálisis de la literatura realizada, evidencia que se debe llevar a cabo más investigaciones direccionadas a la población de adultos mayores de más de 60 años, que puedan ser diagnosticados a través de la tomografía de coherencia óptica de enfermedades de Alzheimer y Parkinson. Aunque existe información sobre el tema, el ahondar más en este procedimiento podría abrir la puerta a un sin fin de biomarcadores que harían posible realizar diagnósticos de forma temprana, con el objeto de optimar la calidad de vida y disminuir el costo a los sistemas de salud que generan los pacientes con EA y EP.

Los autores de este estudio no reportan ningún conflicto de interés.

\section{Recomendaciones}

Luego del análisis realizado en el presente trabajo de investigación, hasta el momento la OCT es un potencial método diagnóstico y en un futuro podría utilizarse como procedimiento coadyuvante en el diagnóstico de las enfermedades neurodegenerativas de Parkinson y Alzheimer por su eficacia y costo más asequible que los biomarcadores de proteínas, en pacientes adultos jóvenes y adultos mayores con factores de riesgo para el desarrollo de estas.

Además, se recomienda la realización de estudios longitudinales multicéntricos con una muestra poblacional mayor, de características más definidas y homogéneas que sea extrapolable para determinar todos los hallazgos posibles en la preclínica de EA y EP, conjuntamente con métodos de medición exactos de las capas de fibras nerviosas de la retina, así también lograr establecer la relación exacta entre los cambios bioquímicos en EA y EP con los cambios estructurales de la retina.

\section{Financiación}

Los autores no tienen ninguna relación económica 


\section{Anexos}

Anexo A: Tabla de análisis de los estudios por lugar de procedencia y año

Autores

1 O' Bryhim, Elizabeth; Kung, Nathan, Coble, Dean7

\section{Revista/Publicación}

Association of Preclinical Alzheimer Disease with Optical Coherence Tomographic Angiography Findings JAMA Network

\section{Título en español}

Asociación de la enfermedad de Alzheimer preclínica con los resultados de la angiografía tomográfica de coherencia óptica
2 Lad, Eleonora; Mukherjee, Dibyendu; Stinnett Sandra; Cousins,

Scott; Potter, G; Burke: Farsiu, Sina; Whistson, Heather9

ELSEVIER

3 Bulut, Mehmet; Kurtulus, Fatma; Gozakaya, Onursal; Erol, Kasim Cengis, Ayse; Melih Akidan; Aylin, Yaman 10 Plos One

4 Maylankody, Pooja; Battu, Rajani; Khanna, Anjani; Lenka, Abhishek; yadavv, Ravi; Kumar, Pramod 11 ELSEVIER

6 Alida van de Kreeke Jacoba; Nguyen, Hoang-Ton; Verbraak, Frank; Visser, Jelle; Pieter 12

Open Access

7 Saute, María; Obis, Javier; Rodrigo, María J; Otin, Sofía; Fuentes, María l; Vilades, Elisai3 Jornal of Ophtalmology

8 Nunes, Ana; Silva, Gilberto, Duque, Cristina; Januario, cristina: Santana, Isabel; Ambrosio, Antonio Francisco Castelo-Branco, Miguel; Bernardes, Ruil 4 Plos One

9 Ahn, Jeeyun; Lee, JeeYoung; Yoon, Jin; Eun; Kim, Jong 15 Related Article

10 Cunha, Provetti; Leonardo; Maciel Almeida, Ana Laura; Costa, Ferreira; Carolina; Monteiro, Mariol 6 Open Access
Evaluation of inner retinal layers as biomarkers in mild cognitive impairment to moderate Alzheimer's disease

Evaluation of optical coherence tomography angiographic findings in Alzheimer's type dementia

Optical coherence tomography as a tool to evaluate retinal changes in Parkinson's disease

Optical coherence tomography angiography in preclinical Alzheimer's disease

Optical Coherence Tomography as a

Biomarker for Diagnosis, Progression, and Prognosis of Neurodegenerative Diseases

Retinal texture biomarkers may help to discriminate between Alzheimer's, Parkinson's, and healthy controls

Retinal thinning associates with nigral dopaminergic loss in de novo Parkinson disease

The role of optical coherence tomography in Alzheimer's disease
Evaluación de las capas internas de la retina como biomarcadores en el deterioro cognitivo leve a la enfermedad de Alzheimer moderada

Evaluación de los hallazgos angiográficos de la tomografía de coherencia óptica en la demencia tipo Alzheimer

La tomografía de coherencia óptica como herramienta para evaluar los cambios retinianos en la enfermedad de Parkinson

Angiografía de tomografía de coherencia óptica en la enfermedad de Alzheimer preclínica

Tomografía de cohe rencia óptica como biomarcador para el diagnóstico, la progresión y el pronóstico de las enfermedades neurodegenerativas

Los biomarcadores de textura retiniana pueden ayudar a discriminar entre Alzheimer, Parkinson y controles sanos

El adelgazamiento de la retina se asocia con pérdida dopaminérgica nigral en la enfermedad de Parkinson de novo

El papel de la tomografía de coherencia optica en la enfermedad de Alzheimer

\section{Conclusiones}

Este estudio sugiere que las personas cognitivamente sanas con EA preclínica tienen anormalidades microvasculares en la retina además de alteraciones arquitectónicas y que estos cambios ocurren en etapas mas tempranas de EA que las que se han demostrado previamente. Se necesitan estudios longitudinales en cohortes de mayor muestra para determinar si este hallazgo es extrapolable para detectar la EA preclínica.

Los cambios en la retina están modestamente relacionados con disminuciones relativas en el rendimiento en una medida de eficiencia de integración audiovisual que se ha avanzado recientemente como un posible marcador cognitivo temprano de deterioro cognitivo leve.

Las áreas de engrosamiento de GCIPL y NFL en la mácula adyacente a las áreas del adelgazamiento sugieren que NFL y GCIPL pueden sufrir cambios dinámicos durante la progresión de EA

En pacientes con EA las patologías vasculares retinianas y coroideas detectadas mediante imágenes de angio-OCT se pueden usar como un nuevo biomarcador en el diagnóstico temprano de la enfermedad, el seguimiento de su progresión y en la investigación de la eficacia de los fármacos.

La densidad de vasos retinianos fue mayor en individuos con EA preclínica.

La técnica de tomografía de coherencia óptica puede permitir el diagnóstico simultaneo de las enfermedades de Alzheimer y Parkinson.

El adelgazamiento de la retina está presente en las primeras etapas de la EP, se correlaciona con la gravedad de la enfermedad y puede estar relacionado con la degeneración dopaminérgica nigral. La imagen retiniana puede ser util para la detección de cambios patológicos que ocurren en la EP temprana. (Nunes, y otros, 2019)

La tecnología OCT debe usarse como un complemento clínico común y muy útil en el diagnóstico y control de los trastornos neurodegenerativos

No se encontró ninguna anormalidad significativa en el grosor de la RNFL en pacientes con EP. La disminución de la CMT en pacientes con EP y una correlación negativa significativa del grosor de RNFL y la CMT con la gravedad de la EP sugieren una remota posibilidad de agotamiento dopaminérgico en la retina. Sin embargo, los estudios a largo plazo están garantizados para validar sud hallazgos. 
Anexo B: Afectaciones oculares presentes en Enfermedad de Alzheimer y Parkinson

\section{MANIFESTACIÓN CLÍNICA EN LA ESTRUCTURAS AFECTADAS ENFERMEDAD DE PARKINSON}



\begin{tabular}{|c|}
\hline \multirow[t]{2}{*}{$\sigma^{+}$} \\
\hline \\
\hline \\
\hline \\
\hline
\end{tabular}

Disminución de la agudeza visual como factor de causante para el progreso hacia alucinaciones visuales.

Alteración de movimientos oculares.

Disminución de la periodicidad de parpadeo.

Déficit de frecuencias espaciales medias

Disminución de la sensibilidad de contraste.

Afectación marcada en el eje rojo-verde, asociados al aumento de los síntomas motores y su empeoramiento.

Desatención altitudinal unilateral izquierda.

Tensiones oculares elevadas, excavaciones, defecto glaucomatoso atribuido a un daño común en la patología de EP y glaucoma.

Disminución de las amplitudes de las ondas alfa y beta, además un valor anormal del coeficiente beta/alfa obtenidos por ERGf

Déficit selectivo a frecuencias espaciales medias.

Disminución en la amplitud y prolongación de latencias por estímulo cromático y luminancia, presentes en el eje azulamarillo.

Afectación marcada de la convergencia.

Exoforia y diplopía

Afectación de los movimientos sacádicos y de seguimiento suave.

Hipometría.

Nistagmo optoquinético anormal con sacudidas.

Movimientos en rueda dentada.

Disminución de la frecuencia de parpade. está intacta.
Déficit de dopamina en la retina

Afectación en el procesamiento de la imagen en las secciones de la corteza temporo-occipital y extraestriada

Las estructuras afectadas son las mismas que en la agudeza visual inclusive esta afectación puede estar disminuida con agudeza visual normal.

Para determinar el daño retiniano se investigó la visión del color mediante la prueba Farnsworth-Munsell 100-hue y los resultados no reflejan la degeneración extranigral.

Afectación de los conos a nivel retinal procesados por la vía parvocelular y koniocelular y la visión acromática por vía magnocelular

Déficit de dopamina en la codificación del espacio visual superior.

Condicionamiento del componente perceptual del hemisferio derecho.

Retraso en el procesamiento de contraste a nivel de la retina.

Importante que la corteza cerebral

A nivel del epitelio pigmentario y fotoreceptores (capa externa de la retina) existe una disfunción bioeléctrica de la vía visual.

Disminución en la actividad eléctrica a nivel de la fóvea.

Trastornos del sistema de seguimiento e insuficiencia en la convergencia.

Alteración sobre el eje progresiva Tritan (prueba Farnsworth-Munsell 100-hue) en donde existe deficiencia del color azul.

Alteraciones sobre el eje: azul-amarillo correspondiente a daño a la retina y sobre el eje rojo verde por daño al nervio óptico.

Pérdida del campo visual por acumulación de $A \beta$.

Disfunción visuoespacial y visuoperceptual

Déficit de la percepción visoespacial.

En pacientes visualmente sintomáticos la estereopsis se encuentra notablemente reducida.

ESTRUCTURAS

AFECTADAS

Atrofia cortical posterior.

Opacidades por acumulación de $\beta$-amiloide en el cristalino.

RETINA

Reducción de células ganglionares en la retina.

Adelgazamiento de la capa de células nerviosas (ganglionares)

\section{NERVIO ÓPTICO}

Disminución a nivel axonal de las células ganglionares.

\section{DISCO ÓPTICO}

Adelgazamiento del borde neurorretiniano.

Palidez de disco.

\section{Atrofia óptica.}

Apraxia oculomotora.

Ataxia óptica.

Agnosia ambiental.

Velocidad retrasada.

Fuente: Journal of neurology, neurosurgery, and psychiatry, Journal of the neurological sciences, The British journal of ophthalmology, Frontiers in neurology, Progress in neuro-psychopharmacology \& biological psychiatry, American journal of Alzheimer's disease and other dementias, Ophthalmic \& physiological optics : the journal of the British College of Ophthalmic Opticians (Optometrists),European journal of neurology, American journal of ophthalmology, Clinical neurophysiology (2020) 


\section{Referencias}

1. Bird TD. Alzheimer Disease Overview. 2a ed. Washington: U.S national Library of Medicine; 2018.

2. The National Institute on Aging, and Reagan Institute Working Group on Diagnostic Criteria for the Neuropathological Assessment of Alzheimer's Disease. Consensus recommendations for the postmortem diagnosis of Alzheimer's disease. Neurobiology of aging. 1997; 18(4 Suppl), S1-S2.

3. Kouli A, Kuan W. Parkison's Disease Pathogenesis And Clinical Aspects. [ebook]. Brisbane: Codon Publications; 2018. Available at: <https:// www.ncbi.nlm.nih.gov/books/NBK536722/> www.ncbi.nlm.nih.gov/bc
[Accessed 13 July 2020].

4. García Martín E, Fuertes Lázaro I, Javier Fernández Tirado F, Emilio Pablo Júlvez L. Utilidad de los nuevos dispositivos de tomografía de coherencia óptica de dominio espectral para el estudio de las demencias degenerativas [Usefulness of the new Spectral-Domain Optical Coherence Tomography (SD-OCT) devices in the study of degenerative dementias]. Arch Soc Esp Oftalmol. 2011 Nov;86(1 1):347-50.

5. Blanks JC, Hinton DR, Sadun AA, Miller CA. Retinal ganglion cell degeneration in Alzheimer's disease. Brain Res. 1989 Nov 6;501 (2):364-72.
6. Cano Osma O. Manifestaciones Oculares y Visuales en el Alzheimer [Homepage en internet]. Castellón de la Plana: School of Advanced Education, Research and Accreditation - SAERA [acceso en 2020 Jul 13]. Disponible en: https://www.saera.eu/manifestaciones-oculares-y-visuales-en-el-alzheimer/

7. O'Bryhim BE, Apte RS, Kung N, Coble D, Van Stavern GP. Association of Preclinical Alzheime Disease With Optical Coherence Tomographic Angiography Findings. JAMA Ophthalmol. 2018 Nov $1 ; 136(11): 1242-1248$

8. Santos CY, Johnson LN, Sinoff SE, Festa EK, Heindel WC, Snyder PJ. Change in retinal structural anatomy during the preclinical stage of Alzheimer's disease. Alzheimers Dement (Amst). 2018 Feb 7;10:196-209.

9. Lad EM, Mukherjee D, Stinnett SS, Cousins SW, Potter GG, Burke JR, et al. Evaluation of inner retinal layers as biomarkers in mild cognitive impairment to moderate Alzheimer's disease. PLoS One. 2018 Feb 8;13(2):e0192646.

10. Bulut M, Kurtulus F, Gözkaya O, Erol MK, Cengiz A, Akıdan M, et al. Evaluation of optical coherence tomography angiographic findings in Alzheimer's type dementia. Br J Ophthalmol. 2018 Feb;102(2):233-237.
11. Mailankody P, Battu R, Khanna A, Lenka A Yadav R, Pal PK. Optical coherence tomography as a tool to evaluate retinal changes in Parkinson's disease. Parkinsonism Relat Disord. 2015 Oct;21 (10):1164-9.

12. van de Kreeke JA, Nguyen HT, Konijnenberg E, Tomassen J, den Braber A, Ten Kate M, et al. Optical coherence tomography angiography in preclinical Alzheimer's disease. Br J Ophthalmol. 2020 Feb;104(2):157-161.

13. Satue M, Obis J, Rodrigo MJ, Otin S, Fuertes MI Vilades $\mathrm{E}$, et al. Optical Coherence Tomography as a Biomarker for Diagnosis, Progression, and Prognosis of Neurodegenerative Diseases. J Ophthalmol. 2016;2016:8503859.

14. Nunes A, Silva G, Duque C, Januário C, Santana I, Ambrósio AF, et al. Retinal texture biomarkers may help to discriminate between Alzheimer's, Parkinson's, and healthy controls. PLoS One. 2019 Jun 21;14(6):e0218826.

15. Ahn J, Lee JY, Kim TW, Yoon EJ, Oh S, Kim YK et al. Retinal thinning associates with nigral do paminergic loss in de novo Parkinson disease. Neurology. 2018 Sep 11;91(11):e1003-e1012.

16. Cunha LP, Almeida AL, Costa-Cunha LV, Costa $\mathrm{CF}$, Monteiro ML. The role of optical coherence tomography in Alzheimer's disease. Int J Retina Vitreous. 2016 Oct 17;2:24. 